\title{
Recent Developments on Airborne Forward Looking Interferometer for the Detection of Wake Vortices
}

\author{
Taumi S. Daniels* \\ NASA Langley Research Center, Hampton, VA, 23681, USA \\ William L. Smith ${ }^{\dagger}$ and Stanislav Kirev ${ }^{\dagger}$ \\ Hampton University, Hampton, VA, 23661, USA
}

\begin{abstract}
A goal of these studies was development of the measurement methods and algorithms necessary to detect wake vortex hazards in real time from either an aircraft or ground-based hyperspectral Fourier Transform Spectrometer (FTS). This paper provides an update on research to model FTS detection of wake vortices. The Terminal Area Simulation System (TASS) was used to generate wake vortex fields of 3-D winds, temperature, and absolute humidity. These fields were input to the Line by Line Radiative Transfer Model (LBLRTM), a hyperspectral radiance model in the infrared, employed for the FTS numerical modeling. An initial set of cases has been analyzed to identify a wake vortex IR signature and signature sensitivities to various state variables. Results from the numerical modeling case studies will be presented. Preliminary results indicated that an imaging IR instrument sensitive to six narrow bands within the 670 to $3150 \mathrm{~cm}^{-1}$ spectral region would be sufficient for wake vortex detection. Noise floor estimates for a recommended instrument are a current research topic.
\end{abstract}

\section{Introduction}

$\mathrm{T}_{\mathrm{a}}^{\mathrm{H}}$ He overall objective of this work was to investigate the feasibility of detecting several types of in-flight aviation hazards with a Forward Looking Interferometer (FLI): clear air turbulence (CAT), wake vortices, dry wind shear, icing, hazardous runway conditions. ${ }^{1}$ The FLI is a 320 x 256 pixel imaging FTS that operates in the IR band $\left(650 \mathrm{~cm}^{-1}\right.$ to $3300 \mathrm{~cm}^{-1}$ wavenumber $){ }^{2}$ The FLI concept combines high spatial, spectral, and radiometric resolution and leveraged remote sensing techniques developed for satellites. ${ }^{3}$ The FLI is an instrument concept that, in the long-term, would obtain in-flight measurements that would alert flight crews to potential weather hazards during all phases of flight. ${ }^{4}$ The research described here was funded by the NASA Aviation Safety program, Atmospheric Environmental Safety Technologies project.

A goal of this study was the development of the measurement methods and algorithms necessary to detect wake vortex hazards in real time from either an aircraft or a ground mounted FLI instrument. The Terminal Area Simulation System (TASS) was used to generate wake vortex fields of 3-D winds, temperature, and absolute humidity. ${ }^{5}$ These fields were input to the Line by Line Radiative Transfer Model (LBLRTM), a hyperspectral radiance model in the infrared, employed for the FTS numerical modeling. ${ }^{6}$

Extensive numerical modeling was conducted using a combination of the two models, TASS and LBLRTM. An initial set of cases was performed to identify wake vortex IR signature sensitivities to various state variables. An algorithm was developed to analyze the three-dimensional data sets that were generated by the combined models. This algorithm selects the FTS channel with the maximum signal, and enables data reduction to two-dimensional imagery. Results from the algorithm are used to determine a wake vortex thermal signature and to simulate FTS imagery.

\footnotetext{
*Aerospace Technologist, MS-473.

${ }^{\dagger}$ Professor, Center for Atmospheric Sciences.
} 


\section{TASS Model Parameters}

For this research, the TASS data sets consisted of three-dimensional grids extracted every 10 seconds after vortex insertion and continuing for a total duration of 60 seconds. Table 1 lists the relevant parameters used by the TASS model.

Table 1. TASS model initialization parameters listed below were used to generate Boeing B747 wake vortices at 10 second intervals after initialization.

\begin{tabular}{ll} 
Parameter & Boeing B747 \\
\hline Grid $(X$ by $Y$ by $Z)$ & $800 \mathrm{~m}$ by $600 \mathrm{~m}$ by $600 \mathrm{~m}$ \\
Initial vortex separation & $50 \mathrm{~m}$ \\
Initial vortex height & $500 \mathrm{~m}$ \\
Initial vortex core radius & $4.5 \mathrm{~m}$ \\
Initial vortex circulation & $565 \mathrm{~m}^{2} / \mathrm{s}$ \\
Initial vortex descent velocity & $1.8 \mathrm{~m} / \mathrm{s}$ \\
Initial temperature $($ at $0 \mathrm{~m})$ & $288.15^{\circ} \mathrm{K}$ \\
Initial pressure (at $0 \mathrm{~m})$ & $1013.25 \mathrm{hPa}$ \\
Temperature lapse rate & $7.4^{\circ} \mathrm{K} / \mathrm{Km}$ \\
Brunt-Vaisala frequency & 0.0015 \\
Eddy dissipation rate & $4 \times 10^{-5} \mathrm{~m}^{2} / \mathrm{s}^{3}$ \\
Water vapor mixing ratio, $Q_{v 0}$ & $6 \mathrm{~g} / \mathrm{kg}$
\end{tabular}

TASS model circulation was determined by the type of aircraft selected and assumed an aircraft with a clean configuration in level flight. Additional parameters are listed in Table 1. In the table, the modeled grid had dimensions $X$, or along track; $Y$, or cross-track; and $Z$, or vertical. This spatial nomenclature was used throughout this research.

\section{IR FTS Numerical Model}

For this modeling effort, the LBLRTM was used to compute radiances within a specified atmosphere of various gases over a specified IR band. ${ }^{7,8}$ The LBLRTM code required input parameters describing atmospheric gas temperatures, pressures, and concentrations. ${ }^{9}$ An optical ray, originating at the top of the atmosphere extended through the TASS modelled wake vortex to the IR FTS instrument mounted on a following-aircraft. Each FTS pixel was simulated as the collected IR energy along a single optical ray.

Each point along the ray was defined by $Z$-axis height, zenith angle to local vertical, and temperature and moisture values of interpolated points embedded in the U. S. Standard Atmosphere. ${ }^{10}$

A list of LBLRTM parameters and ranges of values is given in Table 2. For these simulations, the unapodized spectral resolution was configured as $0.5 \mathrm{~cm}^{-1}$. The output data was apodized using the Beer function ${ }^{11}$ to yield output with various spectral resolutions. For the results presented, the wavenumber spectrum ranged from $650 \mathrm{~cm}^{-1}$ to $3300 \mathrm{~cm}^{-1}$ at 1 and $16 \mathrm{~cm}^{-1}$ intervals. Simulations at lower spectral resolutions were performed but are not presented.

Table 2. All simulations were conducted using the LBLRTM simulation parameters listed here. Typically, only one of these parameters was varied at a time.

\begin{tabular}{ll} 
Parameter & \multicolumn{1}{c}{ Range or Value } \\
\hline Spectral Resolution & $16 \mathrm{~cm}^{-1}$ (apodized) \\
Range of IR Band & $650 \mathrm{~cm}^{-1}$ to $3330 \mathrm{~cm}^{-1}$ \\
Aircraft Altitudes & $0 \mathrm{~km}, 1 \mathrm{~km}, 3 \mathrm{~km}, 6 \mathrm{~km}$ and $10 \mathrm{~km}$ \\
Elevation Angle & $0^{\circ}, 5^{\circ}, 10^{\circ}$, and $15^{\circ}$ \\
Distance to Vortices & $500 \mathrm{~m}$ \\
Wake Vortex Depth & $300 \mathrm{~m}$
\end{tabular}

The altitude of the aircraft and wake vortex was a parameter with values ranging from $10 \mathrm{Km}$ (a typical cruise altitude), $3 \mathrm{~km}$, a typical holding pattern altitude, approach or $1 \mathrm{~km}$, to landing or $0 \mathrm{~km}$. Wake vortices may be persistent at any altitude and can be encountered by other aircraft regardless of flight phase (takeoff, cruise, hold, approach).

The LBLRTM model was configured to use the following atmospheric gases: $\mathrm{H}_{2} \mathrm{O}, \mathrm{CO}_{2}, \mathrm{CO}, \mathrm{NO}_{2}$, $\mathrm{NO}, \mathrm{CH}_{4}$, and $\mathrm{SO}_{2}$. The wake vortex was modeled as a region along a ray of elevated temperature and diminished absolute humidity. This model assumed that a generated vortex pair captured cooler, dryer air from a higher altitude, and descended into a warmer, moister ambient environment. As a worst case scenario, the leading and following aircraft were collinear, such that the vortex pair posed the greatest hazard. In this case, the FTS field of view has rays aligned with the vortex axis. The vortex was $500 \mathrm{~m}$ from the following aircraft and had a depth along the ray of $300 \mathrm{~m}$. The elevation angle corresponded to FTS instrument pointing angles distinct from aircraft angle of attack. 


\section{Maximum Channel Standard Deviation Algorithm}

The following algorithm was devised to reduce both simulated and measured FTS data, though only the simulated data is analyzed in this research. The algorithm performs a statistical comparison of all pixels for a particular wavenumber channel, in order to select that channel with the most signal.

Within each FTS data cube $T_{B}(y, z, \nu)$, a single channel contains an array of brightness temperature data in the two spatial dimensions, $y$ and $z$, and spectral dimension, $\nu$. Let $L$ and $M$ be the number of pixels in the horizontal and vertical dimensions, respectively. Also, let $N$ be the number of channels, or wavenumbers in the spectral dimension. The first step in this algorithm eliminates natural radiance variations due to elevation angle. Brightness temperature deviations removed the row mean using

$$
T_{B}^{*}(i, j, k)=T_{B}(i, j, k)-\frac{1}{M} \sum_{j=1}^{M} T_{B}(i, j, k) .
$$

for $i=[1 \ldots L], j=[1 \ldots M]$, and $k=[1 \ldots N]$. The next step was to compute the spatial mean from the brightness temperature deviations. The spatial mean was defined as

$$
\overline{T_{B}}(k)=\frac{1}{L+M} \sum_{i=1}^{L} \sum_{j=1}^{M} T_{B}^{*}(i, j, k)
$$

The spatial standard deviation for each channel was computed as

$$
\sigma_{Y Z}(k)=\sqrt{\frac{1}{L+M} \sum_{i=1}^{L} \sum_{j=1}^{M}\left[T_{B}^{*}(i, j, k)-\overline{T_{B}}(k)\right]^{2}}
$$

On a per channel basis, the spatial standard deviation was used to define a thermal signature for a wake vortex. Results from simulations that varied altitude are shown in the next section.

The next step was to search for the channel, $\nu_{\max }$, with the maximum standard deviation.

$$
\nu_{\text {max }}=k=\max _{k}^{N}\left[\sigma_{Y Z}(k)\right]
$$

where $N$ was the number of channels and $k$ was wavenumber. By this process, the spatial brightness temperature at $\nu_{\max }$ was selected for each pixel, forming a Maximum Channel Standard Deviation (MCS) image.

$$
\operatorname{MCS}(i, j)=T_{B}^{*}\left(i, j, \nu_{\max }\right)
$$

Results are shown as images of $T_{B}$ at $\nu_{\max }$ for simulated FTS imagery.

\section{Simulation Results}

TASS output for the Boeing B747 wake vortex temperature field is shown in Figure 1 (a) and absolute humidity field in Figure 1 (b). These images are from 3-D data in the $Y Z$ plane at $X=0$. The thermal variation illustrated in the data was about $1^{\circ} \mathrm{K}$, using the color scale for dark red to yellow. This small temperature variation is largely a function of the temperature lapse rate of the atmosphere.

As described in Section III, TASS data was embedded within the Standard Atmosphere. A set of rays through this combination were used to simulate FTS pixels. Each ray corresponded to a single pixel. The geometry of this scheme was parameterized and could be varied to study various effects. The primary variable of interest for this research was altitude.

The output LBLRTM radiance estimates exhibited natural variation unrelated to atmospheric effects. The Planck function was used to remove spectral variability. A typical brightness temperature spectrum for a single ray extending from the top of the atmosphere down to the FTS is shown in Figure 2. For this particular plot, the FTS was simulated at an altitude of $1 \mathrm{~km}$ and with an elevation angle of 5 degrees. The range of temperatures is indicative of the emission spectrum and is too great an amplitude to discern the small variations associated with a wake vortex. 


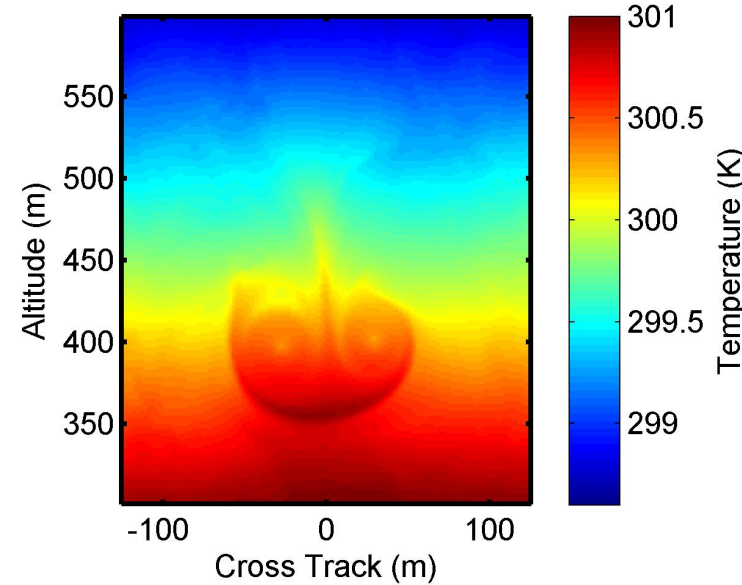

(a) TASS Temperature

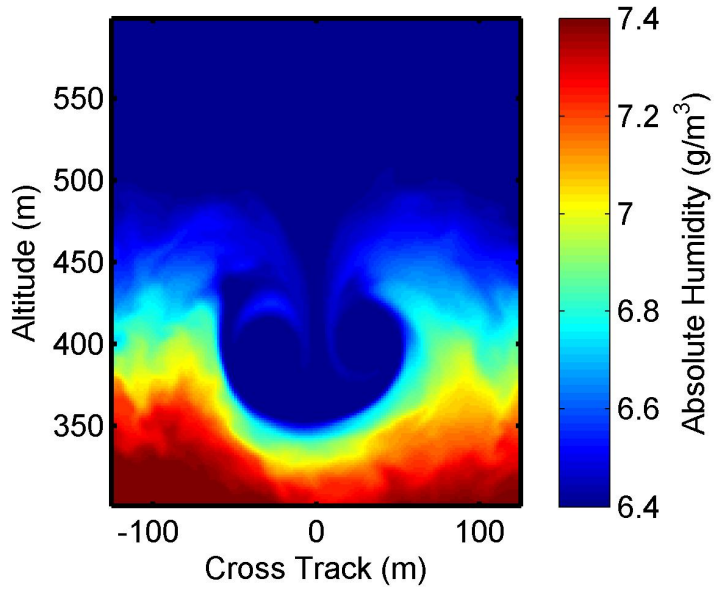

(b) TASS Absolute Humidity

Figure 1. TASS Boeing B747 at $60 \mathrm{~s}$ after initiation showing wake vortex fields.

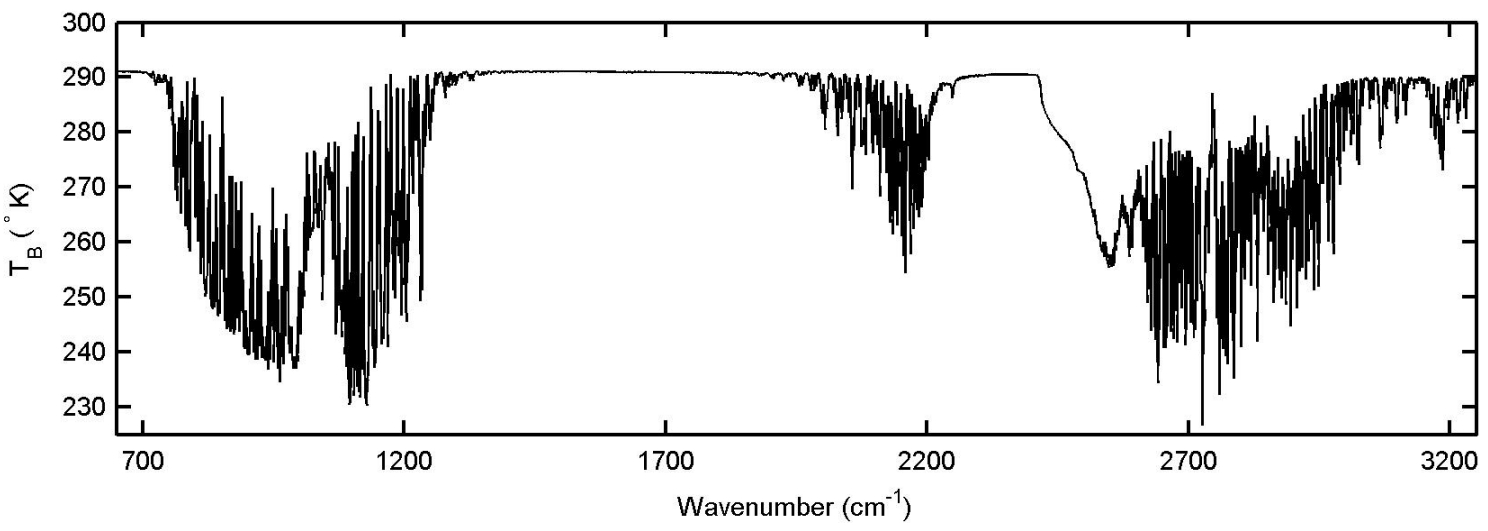

Figure 2. Sample brightness temperature spectrum from LBLRTM at $1 \mathrm{~km}$ altitude and elevation 5 degrees.

The wake vortex thermal signature is illustrated in the following plots of $\sigma_{Y Z}$, shown for FTS simulations with $16 \mathrm{~cm}^{-1}$ spectral resolution. Figure $3(\mathrm{a})$ is at altitude $0 \mathrm{~km}$, Figure $3(\mathrm{~b})$ at $3 \mathrm{~km}$, Figure 3(c) at 6 $\mathrm{km}$, and Figure $3(\mathrm{~d})$ at $10 \mathrm{~km}$. At this lower spectral resolution, the FTS model has smoothed or filtered the higher resolution spectral content. The result is a set of plots that reveal the thermal signature of the wake vortex. In these plots, the wake vortex thermal signature can be clearly defined as consisting of six distinct bands. More importantly, these results illustrate that a high-resolution FTS is not needed, and that an imaging radiometer with at least $16 \mathrm{~cm}^{-1}$ can be used instead.

As shown in Figure 4, the $\sigma_{Y Z}$ data can be used to select wavenumber bands that represent the wake vortex thermal signature. From this plot, the wake vortex thermal signature can be defined as incorporating the following five narrow spectral bands: 670 to $750 \mathrm{~cm}^{-1}, 1250$ to $1450 \mathrm{~cm}^{-1}, 1580$ to $1660 \mathrm{~cm}^{-1}, 1800$ to $2100 \mathrm{~cm}^{-1}$, and 2200 to $2350 \mathrm{~cm}^{-1}$. A sixth band above $3000 \mathrm{~cm}^{-1}$ was excluded due to non-monotonic altitude variation. From the plots of Figure 3, the different IR bands composing the wake vortex thermal signature are seen to exhibit altitude dependence. Bands 1 and 5 are amplitude invariant with altitude, while Bands 2, 3 and 4 merge together above $3 \mathrm{~km}$. Band 6 as evidenced by the appearance of a large spike in Band 6 of Figure 3(d). By utilizing these bands, an imaging IR instrument will have higher sensitivity to the vortex radiative emission and be less sensitive to altitude dependencies exhibited in the plots. The specific bands with optimal sensitivity depend upon altitude and atmospheric moisture concentration.

Results from the MCS algorithm, as defined in Section IV, are presented. A simulated FTS image plot with resolution $16 \mathrm{~cm}^{-1}$ at altitude $0 \mathrm{~km}$ is shown in Figure $5(\mathrm{a})$ and at altitude $3 \mathrm{~km}$ in Figure $5(\mathrm{~b})$. In 


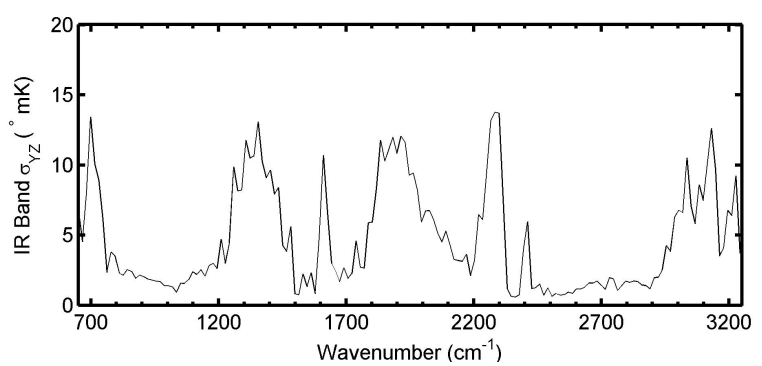

(a) $0 \mathrm{~km}$ altitude

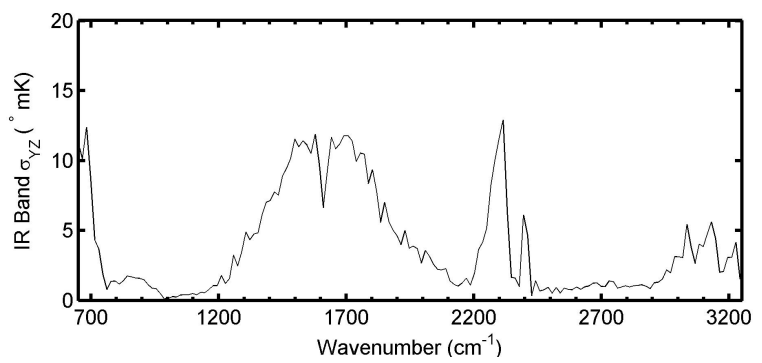

(c) $6 \mathrm{~km}$ altitude

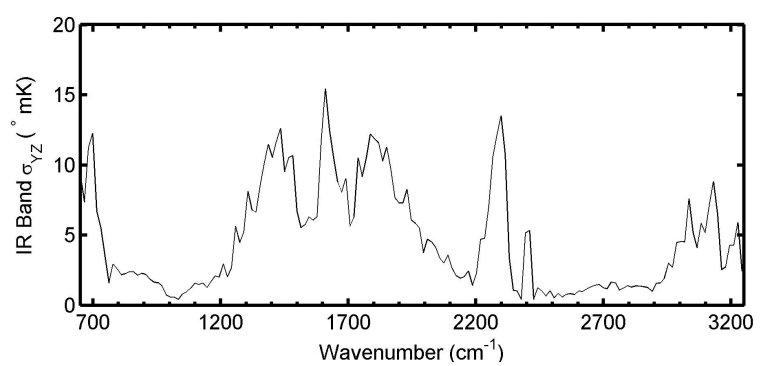

(b) $3 \mathrm{~km}$ altitude

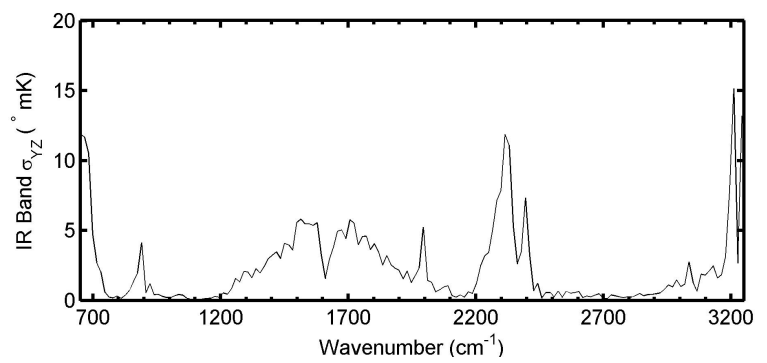

(d) $10 \mathrm{~km}$ altitude

Figure 3. $\mathbf{T}_{B} \sigma_{Y Z}$ at $16 \mathrm{~cm}^{-1}$ resolution.

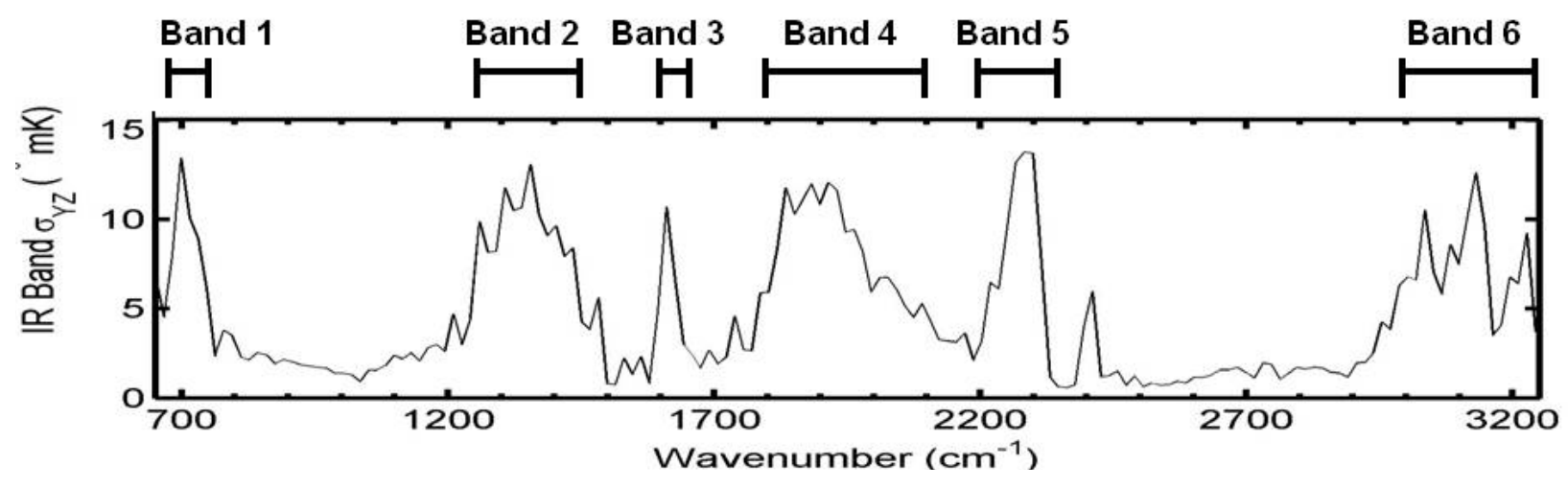

Figure 4. Illustration of wake vortex thermal signature with identification of six IR bands.

these imagery plots, a single channel represent the entire spectrum at each pixel. This channel is selected using the MCS algorithm. Note that the wake vortex pair is evidenced by semicircle of warm, moist air. Due to long computation times for the large number of rays, subsampling of the original TASS data results in the pixelation seen in these figures. The temperature range for these plots is about $0.025^{\circ} \mathrm{K}$ using the color scale for dark red to yellow. The variation does not degrade significantly with altitude.

A second set of FTS images is shown in the plots of Figure 6(a) at altitude $6 \mathrm{~km}$ and in Figure 6(b) at altitude $10 \mathrm{~km}$. As with the previous plots at the lower altitudes, the wake vortices are evident. However, these plots illustrate a loss of signal amplitude with increasing altitude. These plots indicate that a thermal signature associated with wake vortices is theoretically capable of being detected by an imaging IR instrument.

\section{Conclusion}

The LBLRTM was employed to model FTS responses to atmospheric variations. Several parameterization cases were performed to identify wake vortex IR signature sensitivities to various state variables. These variables included temperature, water vapor, atmospheric gas, turbine jet exhaust gas concentration, altitude, 


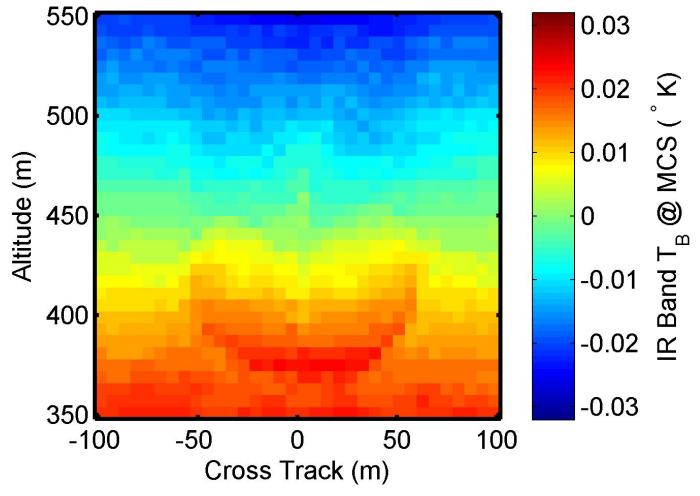

(a) Channel $698 \mathrm{~cm}^{-1}$ at altitude $0 \mathrm{~km}$

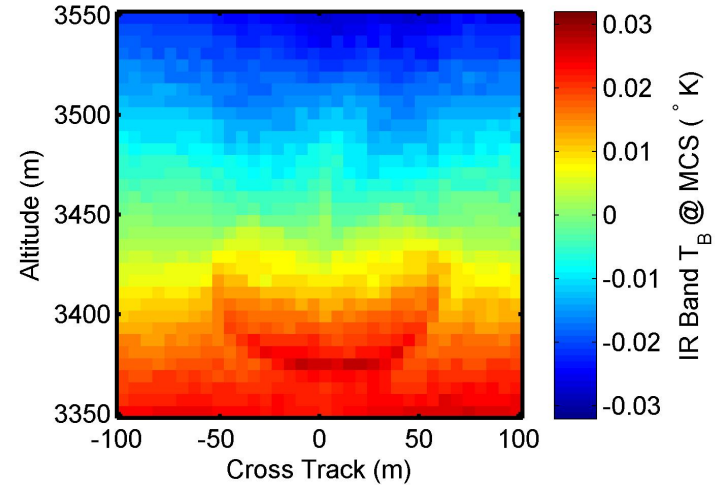

(b) Channel $1610 \mathrm{~cm}^{-1}$ at altitude $3 \mathrm{~km}$

Figure 5. FTS simulations at resolution $16 \mathrm{~cm}^{-1}$ for IR Band $650 \mathrm{~cm}^{-1}$ to $3350 \mathrm{~cm}^{-1}$ at elevation angle $5^{\circ}$.

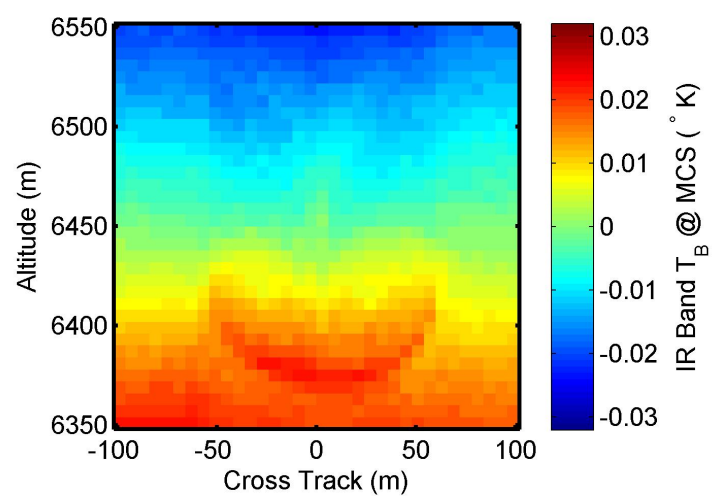

(a) Channel $1675 \mathrm{~cm}^{-1}$ at altitude $6 \mathrm{~km}$

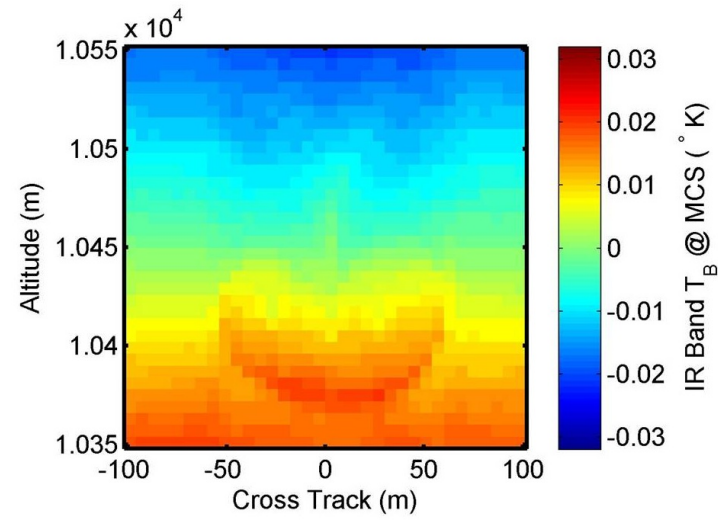

(b) Channel $668 \mathrm{~cm}^{-1}$ at altitude $10 \mathrm{~km}$

Figure 6. FTS simulations at resolution $16 \mathrm{~cm}^{-1}$ for IR Band $650 \mathrm{~cm}^{-1}$ to $3350 \mathrm{~cm}^{-1}$ at elevation angle $5^{\circ}$.

viewing angle or elevation, and distance from FTS model to wake vortex.

The results of this preliminary study revealed that a high resolution FTS-type instrument may not be needed to detect a wake vortex thermal spectral signature. Additional simulations are needed to examine instrument response to wake vortex distance and resulting imagery generated from the $\sigma_{Y Z}$ data.

On a per channel basis, the spatial standard deviation was used to define a thermal signature for a wake vortex. The primary variable of interest for this research was altitude. Results from simulations that varied distance are a future research topic. For the simulated wake vortices, the wake vortex thermal signature was clearly visible in the resulting imagery.

In these idealized simulations, no instrument noise was modeled. As such, a required noise floor needs to be estimated. This necessary step requires the simulation of FTS or radiometer imagery. In addition, the spectral response of the IR sensor was not simulated. Typical IR imaging FTS designs employ either $\mathrm{HgCdTe}$ or InSb focal plane arrays as the IR sensor, each having an associated responsivity spectral band. Unfortunately, this research identified five narrow bands that extend across both of these two different IR sensor types.

Based on this research, a recommended instrument type for wake vortex detection is an imaging IR radiometer sensitive in the following five narrow spectral bands: 670 to $750 \mathrm{~cm}^{-1}, 1250$ to $1450 \mathrm{~cm}^{-1}, 1580$ to $1660 \mathrm{~cm}^{-1}, 1800$ to $2100 \mathrm{~cm}^{-1}$, and 2200 to $2350 \mathrm{~cm}^{-1}$. Further research is needed to determine a recommended a maximum instrument noise floor after additional simulations are performed. After altitude, the next major variable of interest is change in distance from the wake vortex. Yet more analysis is required to compare these simulated results with measured data.

Additional research is needed to analyze the impact of turbine jet exhaust on these results. How will 
rolled-up exhaust gas change the thermal wake vortex signature for these radiative transfer simulations? Can the IR chemical spectroscopic signal of turbine jet exhaust be used as a proxy for wake vortex detection? The numerical techniques describe here should enable the research to resolve these questions.

\section{References}

${ }^{1}$ Smith, Sr.., W. L., Kireev, S., Gimmestad, G., West, L., Liu, X., Schaffner, P., and Murray, J. J., "Airborne ForwardLooking Interferometer Turbulence Investigation," Fourier Transform Spectroscopy/ Hyperspectral Imaging and Sounding of the Environment, OSA Technical Digest Series (CD), No. FThB2, Optical Society of America, Feb. 2007.

${ }^{2}$ West, L. L., Gimmestad, G. G., Smith, Sr., W. L., Kireev, S., Cornman, L., Schaffner, P., and Tsoucalas, G., "Applications of a Forward-Looking Interferometer for the On-board Detection of Aviation Weather Hazards," Tech. Rep. NASA/TP-2008215536, NASA, Langley Research Center, October 2008.

${ }^{3}$ Smith, Sr., W. L., Kireev, S., West, L. L., Gimmestad, G. G., Cornman, L., Feltz, W., Perram, G., and Daniels, T., "Interferometric radiometer for in-flight detection of aviation hazards," Remote Sensing Applications for Aviation Weather Hazard Detection and Decision Support, edited by W. F. Feltz and J. J. Murray, Vol. 7088, 2008.

${ }^{4}$ West, L. L., Gimmestad, G. G., Herkert, R., Smith, Sr., W. L., Kireev, S., Schaffner, P., Daniels, T., Cornman, L., Sharman, R., Weekley, A., Perram, G., Gross, K., Smith, G., Feltz, W., Taylor, J., and Olson, E., "Hazard Detection Analysis for a Forward-Looking Interferometer," Tech. Rep. NASA/TP-2010-216845, NASA, Langley Research Center, August 2010.

${ }^{5}$ Proctor, F. H., "The Terminal Area Simulations System, Volume 1: Theoretical Formulation," 1987, NASA CR-4046.

${ }^{6}$ Smith, Sr., W. L., West, L. L., Gimmestad, G. G., and Lane, S. E., "Ultra-spectral measurements of surface emissivity with an imaging interferometer spectrometer," Hyperspectral Imaging and Sounding of the Environment, OSA Technical Digest (CD), No. HTuC4, Optical Society of America, July 2011.

${ }^{7}$ Clough, S. A., Iacono, M. J., and Moncet, J.-L., "Line-by-Line Calculations of Atmospheric Fluxes and Cooling Rates: Application to Water Vapor," Journal of Geophysical Research, Vol. 97, No. D14, 1992, pp. 15761-15785.

${ }^{8}$ Clough, S. A. and Iacono, M. J., "Line-by-line calculation of atmospheric fluxes and cooling rates 2. Application to carbon dioxide, ozone, methane, nitrous oxide and the halocarbons," Journal of Geophysical Research, Vol. 100, No. D8, 1995, pp. 16519-16535.

${ }^{9}$ Shephard, M., Cady-Pereira, K., and Rieu-Isaacs, H., "LBLRTM, LNFL, and Line Parameter Databases," [Software]. Retrieved from http://rtweb.aer.com/, May 2011.

${ }^{10}$ COESA, "U.S. Standard Atmosphere, 1976," Tech. Rep. NOAA-S/T 76-1562, NOAA, Oct. 1976.

${ }^{11}$ Norton, R. H. and Beer, R., "New apodizing functions for Fourier spectrometry," Journal of the Optical Society of America, Vol. 66, No. 3, March 1976, pp. 259-264. 